\title{
Common and Rare Histological Variants of Hepatoblastoma in Children: A Pathological Diagnosis and Review of the Literature
}

\author{
Sushma Bharti ${ }^{a}$ Jyotsna Naresh Bharti ${ }^{a}$ Arvind Sinha ${ }^{b}$ Taruna Yadav $^{c}$ \\ aDepartment of Pathology, All India Institute of Medical Sciences, Jodhpur, India; 'bepartment of Pediatric Surgery, \\ All India Institute of Medical Sciences, Jodhpur, India; 'Department of Radiology, All India Institute of Medical \\ Sciences, Jodhpur, India
}

\section{Keywords}

Hepatoblastoma $\cdot$ Mixed type $\cdot$ Teratoid $\cdot$ Small-cell variant . Children

\section{Abstract \\ Hepatoblastoma (HB) is a rare tumor, but it is the most com- mon primary liver malignancy in children and comprised of approximately $1 \%$ of all pediatric malignancies. Mostly, this tumor is sporadic in nature but can show a syndrome asso- ciation. Upregulation in $\mathrm{Wnt} / \mathrm{\beta}$-catenin pathway can be there in $70-80 \%$ cases of HB. Most often present as abdomi- nal mass and has a raised alpha-fetoprotein levels. Distant metastasis usually occurs in the lungs. HB is classified into 2 broad categories: epithelial and mesenchymal type. The ma- jority of $\mathrm{HB}$ are epithelial type. The HB must be distinguished from focal nodular hyperplasia, hepatocellular adenoma, and hepatocellular carcinoma, while small-cell undifferenti- ated HB from the malignant rhabdoid tumor. The histomor- phology and immunohistochemistry are essential for the di- agnosis of different HB. The neoadjuvant chemotherapy fol-}

lowed by surgery is the mainstay of the treatment. The 2 well-established prognostic factors of $\mathrm{HB}$ are stage and histological type. Herein, we report a case series of common and rare histological variants of HB.

(C) 2021 The Author(s)

Published by S. Karger AG, Basel

\section{Introduction}

Hepatoblastoma (HB) is a rare, but commonest primary malignant liver tumor in children accounting for approximately $1 \%$ of all pediatric malignancies, commonly seen in $<5$ years of age, followed by hepatocellular carcinoma and sarcoma [1]. In adults, HB is rare and aggressive neoplasm with a poor prognosis. The etiological factors and pathogenesis in adults differ from childhood. The annual incidence of $\mathrm{HB}$ is about 1-2 per 10,000 children in $<15$ years of age. Here, we present a case series of HB in children with different histomorphology.

karger@karger.com www.karger.com/gat

Karger $\stackrel{\text { ' }}{5}$

GOPEN ACCESS
(C) 2021 The Author(s)

Published by S. Karger AG, Basel

This article is licensed under the Creative Commons AttributionNonCommercial-NoDerivatives 4.0 International License (CC BYNC-ND) (http://www.karger.com/Services/OpenAccessLicense) Usage and distribution for commercial purposes as well as any distribution of modified material requires written permission.
Department of Pathology, All India Institute of Medical Sciences Basni Phase II

Jodhpur 342005 (India)

Jyotsnamamc@gmail.com 


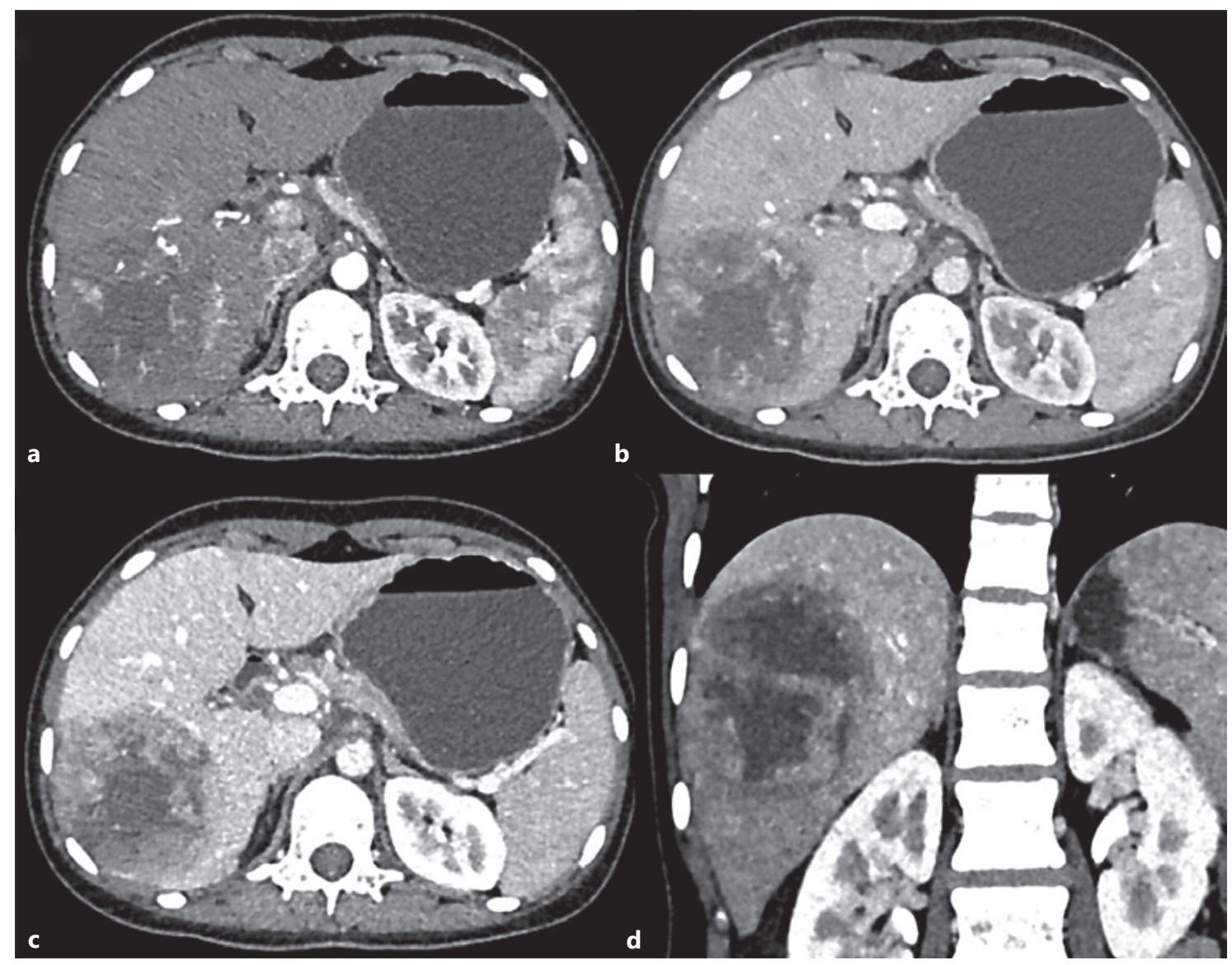

Fig. 1. CASE 1: Triple-phase contrast-enhanced CT axial (a-c) and coronal (d) images: in arterial phase, mass in right lobe shows peripheral arterial enhancement (a), portal venous (b), hepatic venous phases - progressive enhancement is seen in the periphery of the tumor with cystic nonenhancing areas in the center of the tumor $(\mathbf{c}, \mathbf{d})$. The rest of the liver shows normal enhancement.

\section{Case 1}

A 2-year-old boy presented in the pediatric surgery outpatient department with complaints of loss of appetite, lethargy, and enlarged abdomen for 2 months. There was a huge abdominal lump over the right hypochondrium. There was a large nodule in imaging replacing most of the right lobe of the liver (Fig. 1). The bilateral lungs were unremarkable, and the liver function test was normal. The coagulation profile was abnormal with raised serum AFP $(>1,000 \mathrm{ng} / \mathrm{mL})$ levels. Liver biopsy showed features of HB. The staging was Pre-Treatment Extent 2 (PRETEXT): segments 5 and 6 and standard risk. The patient was given cisplatin monotherapy before surgery. The patient was operated and we received right lobectomy measuring $14 \times 7 \times 5.5 \mathrm{~cm}$, weighing $226 \mathrm{gm}$. Externally, it showed few congested areas and a nodule measuring $1 \mathrm{~cm}$ in diameter. Cut surface shows variegated appearance (Fig. 2a). The tumor was abutting liver capsule with the distance measuring 0.1 $\mathrm{cm}$. Tumor measured $6.9 \times 6.4 \times 4.5 \mathrm{~cm}$. Gall bladder and resection margins were free from tumor. On microscopy, a capsulated tumor was noted, arranged in sheets and trabeculae. The epithelial component was admixed with the mesenchymal component. Many heterologous elements in variable proportion were noted like melanin pigment, abundant glial tissue, pacinian corpuscles, and areas of osteoid and cartilage formation along with areas of necrosis (Fig. 2b-d). There was no capsular invasion, lymphovascular emboli, and extramedullary hematopoiesis. The final diagnosis was mixed $\mathrm{HB}$ with teratoid features.

\section{Case 2}

A 2-year-old boy presented to the pediatric surgery outpatient department with similar complaints and findings as the previous case. Bilateral lung, liver function test, and coagulation profile were normal with raised serum AFP $(>2,00,000 \mathrm{ng} / \mathrm{mL})$. The staging was PRETEXT 2 (segments 7 and 8) and standard risk. The patient was given cisplatin monotherapy 2 cycles before surgery. We received a lobe hepatectomy specimen along with a gall bladder. There was an ill-defined nodule measuring $5 \times 4 \times 3 \mathrm{~cm}$ noted 

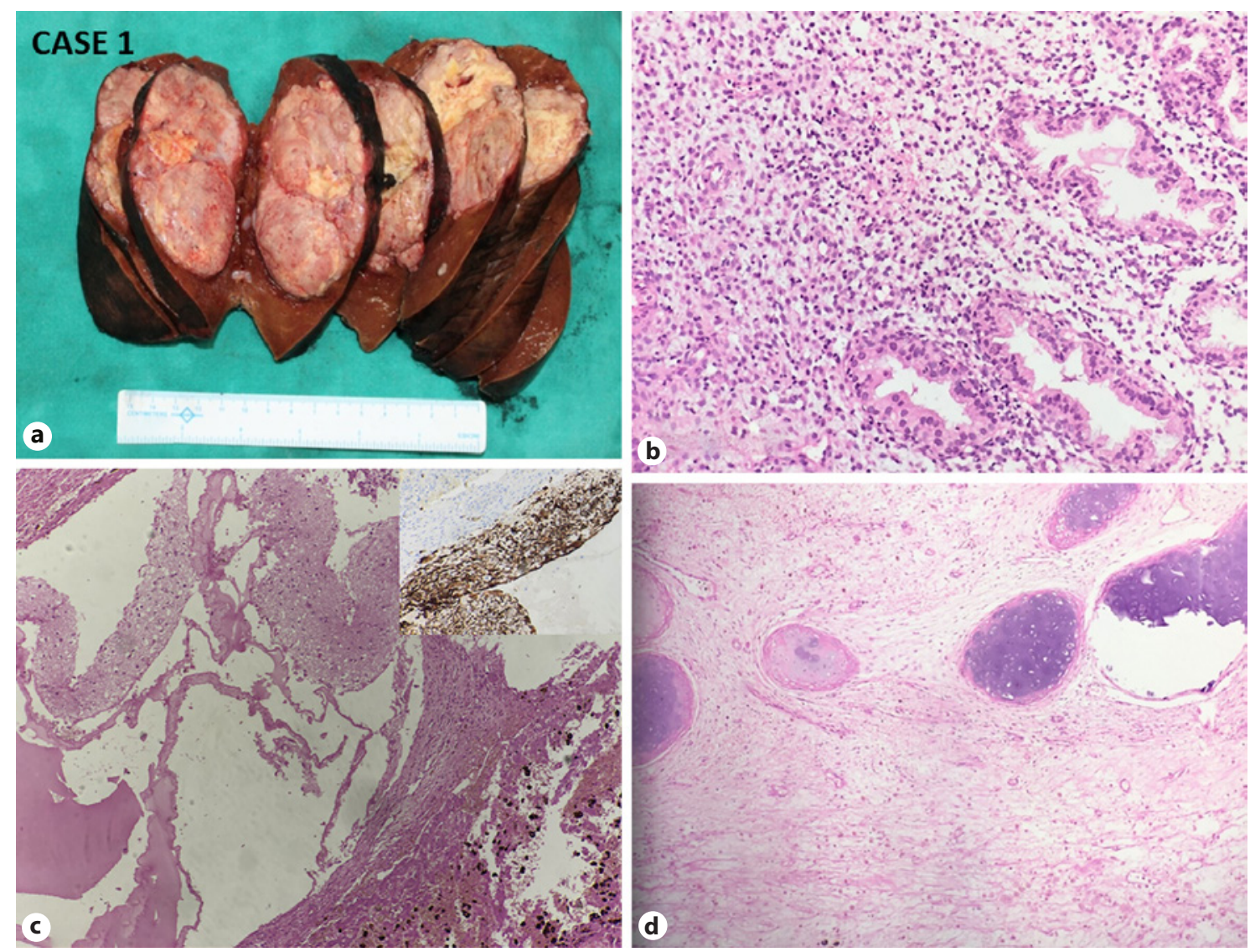

Fig. 2. CASE 1: Mixed HB with the teratoid feature. a Gross: well-defined tumor nodule, abutting the capsular surface $(\mathrm{H}$ and $\mathrm{E}, \times 200)$. b Microphotograph shows an epithelial component of HB. c Microphotograph shows Glial component and melanin pigment ( $\mathrm{H}$ and $\mathrm{E}, \times 200)$ (right upper inset showing GFAP immunopositivity). d Microphotograph shows osteoid and cartilage formation $\mathrm{H}$ and E, $\times 200$ ). HB, hepatoblastoma.

with areas of hemorrhage and necrosis (Fig. 3a). The tumor nodule is $1.5 \mathrm{~cm}$ away from resection surface. On histopathology, a wellcircumscribed tumor was arranged in sheets, nodules, and trabeculae. The epithelial component was admixed with the mesenchymal component (Fig. 3b). The large areas of osteoid formation, fibrosis, calcification, necrosis, and hemorrhage were noted with no evidence of teratoid features, extramedullary hematopoiesis, and lymphovascular emboli. The liver resection margin and gall bladder were free of tumor. The final diagnosis of mixed HB was made.

\section{Case 3}

A 1-year-old male child came to the pediatric surgery outpatient department with complaints of abdominal distension and mass per abdomen in the right upper quadrant. CT abdomen suggests a large lobulated solid cystic lesion involving the liver entire right lobe with focal rupture into the subcapsular spaces. AFP level was normal $(3.03 \mathrm{IU} / \mathrm{mL})$. The patient had hyperbilirubinemia.
The staging was PRETEXT 2 (segments 5, 6, 7, and 8-right lobe) and high risk. We received fragmented liver biopsy showing tumor cells arranged in solid sheets of dyscohesive small blue cells having a small round nucleus, fine chromatin, and indistinct nucleolus, and fine granular scant eosinophilic cytoplasm. Occasional singlecell apoptosis and mitotic activity were noted. Some tumor cells were arranged around the vessels (Fig. 4a). No mesenchymal or teratoid or rhabdoid components were noted in the biopsy. The closest differential was a malignant rhabdoid tumor and other small blue round cell tumors. On immunohistochemistry, the tumor cells were immunoreactive for cytokeratin, vimentin, and focally positive for alpha-fetoprotein (Fig. $4 \mathrm{~b}-\mathrm{d}$ ). There was a loss of INI-1, and Ki-67-40\%. Tumor cells were negative for CD99, Glypican 3, Myo-D, Desmin, LCA, NSE, and CD56. This was, hence, reported as INI-1 negative, Small-cell undifferentiated variant of HB (SCUD-HB).

The patient was given 3 cycles of PLADO, that is, Doxorubicin and cisplatin (75\%). The post-chemotherapy AFP level was normal $(3.01 \mathrm{IU} / \mathrm{mL})$. The patient is doing well at 5 months of follow-up. 

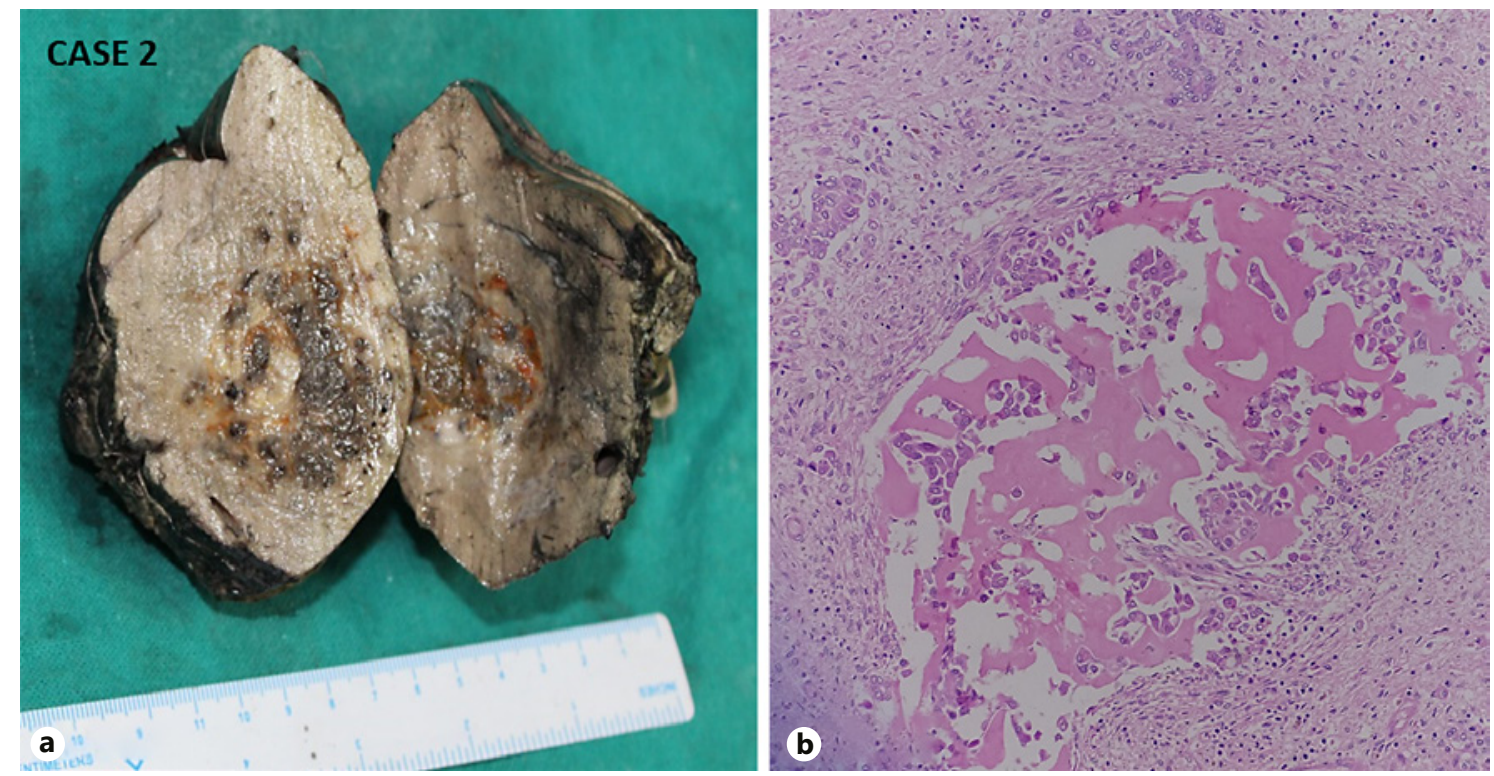

Fig. 3. CASE 2: Mixed hepatoblastoma. a Gross: a tumor shows a variegated cut surface. b Microphotograph shows an epithelial component admixed with the mesenchymal component $(\mathrm{H}$ and $\mathrm{E}, \times 200)$.
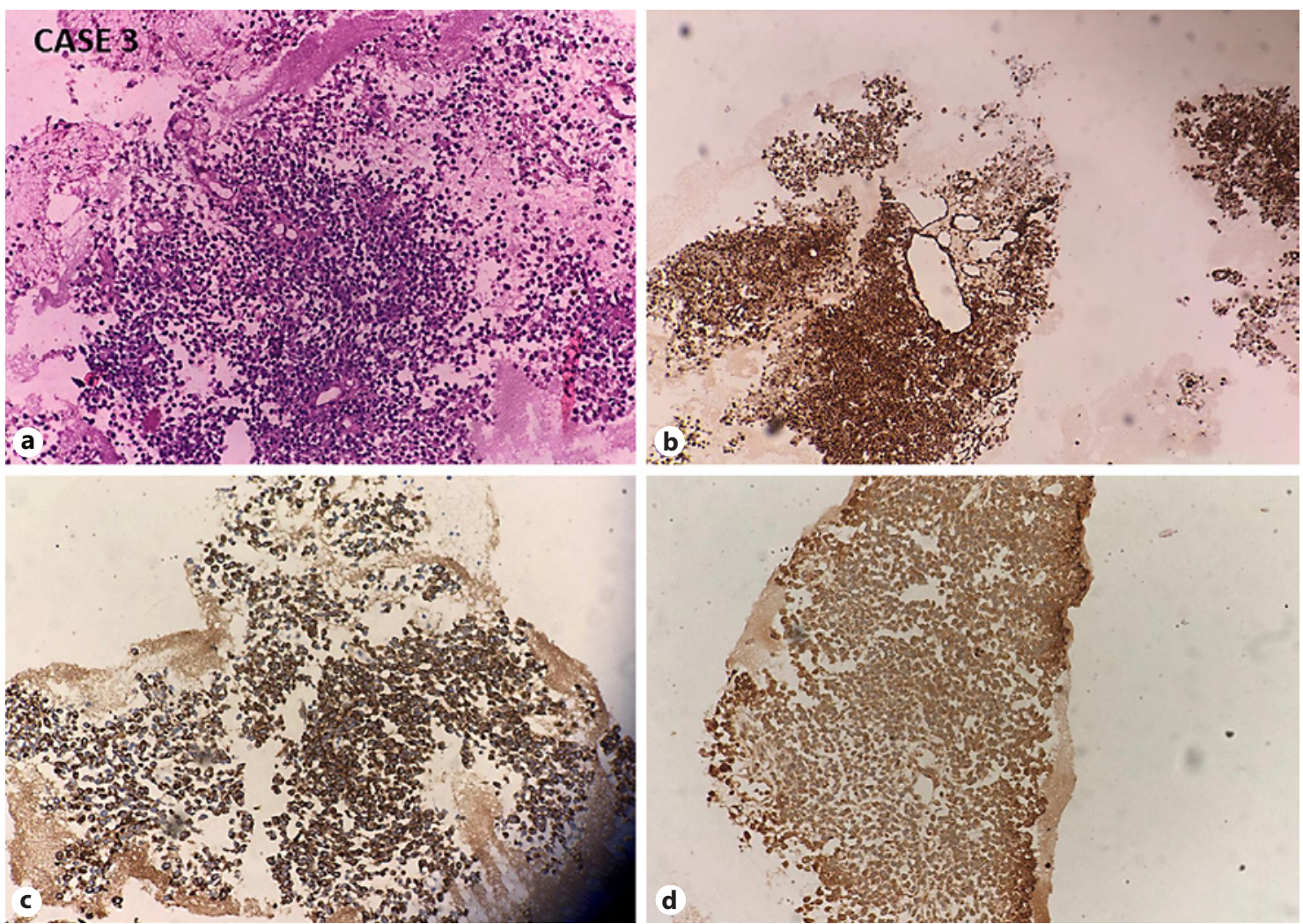

Fig. 4. CASE 3: Small-cell undifferentiated HB. a Microphotograph shows sheets and perivascular arrangement of dyscohesive small blue cells with scant cytoplasm $(\mathrm{H}$ and E, $\times 200)$. Tumor cells are immunoreactive for cytokeratin (b), vimentin (c), and focally for alpha-fetoprotein (d). HB, hepatoblastoma. 


\section{Discussion}

Any congenital anomalies, tumors, trauma, infection, abscess, or any organ enlargement can cause abdominal masses in infants and children [1]. Therefore, before planning any invasive procedures, age, and abdominal ultrasonography is necessary, as age significantly affects the differential diagnosis and radiological imaging aids in the organ's exact localization. The commonest abdominal tumors in children are neuroblastoma and wilms' tumor, and less common are germ cell tumors and rhabdomyosarcoma. $90 \%$ of neuroblastoma are diagnosed by the age of 8 . At the same time, most of the wilms' tumor is diagnosed before the mean age of 3 years. Although the liver mass is uncommon in infants and children, comprising $15 \%$ of all primary abdominal tumors, two-third are malignant, and $\mathrm{HB}$ is the most common one [2]. HB is commonly seen in males as solitary liver mass (80-85\%), often affecting the right lobe of the liver.

Mostly, this tumor is sporadic but can show a syndromic association, seen in 5\% cases [1]. Parental exposure to metals, birth pills during pregnancy, and very low birth weight baby increases its risk. The exact pathogenesis is still unknown, but it is thought to originate due to dysregulation of the primitive hepatic stem cells (HB) during the embryogenesis that contributes to malignant transformation. The most frequent genetic alteration is upregulation of the $\mathrm{Wnt} / \beta$-catenin signaling pathway, seen in $70-90 \%$ cases of HB [3]. The most typical presentation is abdominal mass, pain, and nonspecific symptoms. $>90 \%$ of patients have raised AFP level except in case of aggressive course and SCUD in which level will be low or normal [4]. AFP is the hallmark of active disease, and it has a direct relation with the size of $\mathrm{HB}$ [5]. Therefore, it is used as a marker of disease activity and monitors therapy response. It is associated with a good prognosis if there is a sudden fall in its level after therapy [6]. However, it is not specific. Jaundice is rare, and LFT is usually normal in HB. The presence of jaundice with a liver mass in pediatric patients rule out HB's possibility and more often seen in biliary rhabdomyosarcoma and undifferentiated sarcoma of the liver [7]. Distant metastasis was almost always seen in the lungs. The risk for metastasis is higher in adults. Anemia and thrombocytosis sometimes present as paraneoplastic syndromes [8].

$\mathrm{HB}$ is classified histologically into epithelial and mixed types and described by Ishak and Glunz [9]. The majority are epithelial, with a variable amount of embryonal and fetal cell morphology similar to the developing liver. The mixed type contains epithelial and neoplastic mesenchy-

Hepatoblastoma and Its Varied

Histological Variants mal elements or shows bone, cartilage, fibrous tissue, and skeletal muscle differentiation. In the present case, mixed HB shows the presence of bone, cartilage, and fibrous tissue. Ahn et al. [10] had reviewed 21 cases of $\mathrm{HB}$ and found that mixed type is commoner than pure epithelial type. Rarely mixed tumors classified as $\mathrm{HB}$ with teratoid features show the variable amount of other heterologous elements in different proportions, such as stratified squamous, mucinous, and ductular cholangioblastic epithelium, immature neuroepithelium, neuroendocrine differentiation, and melanin pigment. Manivel et al. first described this category [2]. There was one mixed-type $\mathrm{HB}$ with teratoid features in the present study representing different heterologous elements in various proportions like melanin pigment and a large area of glial tissue, osteoid formation, and pacinian corpuscles (Fig. 4). The differential diagnosis of focal nodular hyperplasia, hepatocellular adenoma, and hepatocellular carcinoma is considered. CD34 and beta-catenin are useful markers for the diagnosis of HB. CD34 shows diffuse capillarization in $\mathrm{HB}$, which is not seen in benign lesions and normal hepatocytes, indicating that sinusoids are lined by endothelial cells in the tumor. Beta-catenin gives nuclear and cytoplasmic positivity in both $\mathrm{HB}$ and normal hepatocytes, which has diagnostic and prognostic significance in $\mathrm{HB}$ [11]. The SCUD subtype shows the variable expression for PanCK, CK8, CK-18, and vimentin. They rarely express AFP and glypican [4]. Because of the heterogeneity of the tumor, its behavior is unpredicted. Therefore, core biopsy and inadequate resection specimens frequently fail to represent all the components of HB. Even a small focus of SCUD can affect the prognosis of the disease [4]. In the present case series, also one case was of INI-1 negative SCUD in a 1-year-old male child. Its closest differential was a malignant rhabdoid tumor, which is morphologically identified as a unique tumor type based on its characteristic "rhabdoid" cytologic features with abundant eosinophilic cytoplasm and eccentric nuclei. However, in the present case, the tumor cells were positive for cytokeratin, vimentin, and focally for alpha-fetoprotein, and $\mathrm{Ki}$ 67-40\%. Tumor cells were negative for CD99, glypican 3, Myo-D, Desmin, LCA, NSE, and CD56. The 2 well-established prognostic factors of $\mathrm{HB}$ are stage, and histological type of HB. The histological types are fetal, embryonal, combined, macrotrabecular, and SCUD. Different subtypes correlate with patient prognosis. SCUD has the worst, and fetal type has a better outcome than embryonal type. Staging is done using the children's oncology group staging system, before any therapy. The Children's Hepatic tumors International Collaboration 
concluded that the advanced PRETEXT stage, presence of portal or hepatic venous involvement, extrahepatic disease, tumor multifocality, metastases, and tumor rupture at presentation, $\geq 8$ years old, and AFP $<100 \mathrm{ng} / \mathrm{mL}$ were associated with a worse outcome [12]. The mainstay of the treatment of HB is adjuvant chemotherapy, followed by complete surgical resection [8]. The overall prognosis of $\mathrm{HB}$ has dramatically improved over the past 30 years. For detecting lung metastasis, CT scan is the most reliable diagnostic modality [13].

\section{Conclusion}

Considering the patient's age, exact location, and nature of the lump, the HB shall be considered as differential diagnosis for abdominal mass in children. Extensive grossing and meticulous examination are very important for the correct diagnosis of $\mathrm{HB}$ and its various components as histological types, which decide the patient's prognosis.

\section{Acknowledgements}

I would like to thank all those who helped in grossing and writing this article.

\section{Statement of Ethics}

Patients have given written informed consent for the publication of the data and images.

\section{Conflict of Interest Statement}

There is no conflict of interest regarding the publication of this article.

\section{Funding Sources}

The authors did not receive any funding.

\section{Author Contributions}

Dr. Sushma Bharti, Senior Resident: written the manuscript and searched the articles. Dr. Jyotsna Naresh Bharti, Associate Professor: diagnosed the rare case, done the manuscript's editing, and provided gross and microscopy images. Dr. Arvind Sinha, Professor, and Head: he was the operating surgeon and provided the clinical details. Dr. Taruna Yadav, Assistant Professor: she provided the radiological images.

\section{References}

1 Davidoff A. Pediatric abdominal masses. In: Myers JA, Millikan KW, Saclarides TJ, editors. Common surgical diseases. New York, NY: Springer; 2008.

2 Manivel C, Wick MR, Abenoza P, Dehner LP. Teratoid hepatoblastoma: the nosologic dilemma of solid embryonic neoplasms of childhood. Cancer. 1986;57(11):2168-74.

3 Takayasu H, Horie H, Hiyama E, Matsunaga T, Hayashi Y, Watanabe Y, et al. Frequent deletions and mutations of the beta-catenin gene are associated with overexpression of cyclin D1 and fibronectin and poorly differentiated histology in childhood hepatoblastoma. Clin Cancer Res. 2001;7(4):901-8.

4 Saxena R, Quaglia A. Hepatoblastoma. WHO classification of tumors of the digestive system. 5th ed; 2020. p. 240-4.

5 Sharma D, Subbarao G, Saxena R. Hepatoblastoma. Semin Diagn Pathol. 2017;34(2): 192-200.
6 Pateva IB, Egler RA, Stearns DS. Hepatoblastoma in an 11-year-old: case report and a review of the literature. Medicine. 2017;96(2): e5858.

7 Fernandez-Pineda I, Cabello-Laureano R. Differential diagnosis and management of liver tumors in infants. World J Hepatol. 2014; 6(7):486-95.

8 Zaman MB. The liver and spleen. In: Koss LG, Melamed MR, editors. Koss' diagnostic cytology and its histopathologic bases. 5th ed. New York: Lippincott Williams \& Wilkins; 2006; p. 1408-10.

9 Ishak KG, Glunz PR. Hepatoblastoma and hepatocarcinoma in infancy and childhood. Report of 47 cases. Cancer. 1967;20(3):396-422.
10 Ahn HJ, Kwon KW, Choi YJ, Kim HJ, Hong SP, Oh D, et al. Mixed hepatoblastoma in an adult: a case report and literature review. J Korean Med Sci. 1997;12:369-73.

11 Bera G, Das RN, Roy P, Ghosh R, Islam N, Mishra PK, et al. Utility of PAS and $\beta$-catenin staining in histological categorisation and prediction of prognosis of hepatoblastomas. Pediatr Surg Int. 2017;33(9):961-70.

12 Czauderna P, Haeberle B, Hiyama E, Rangaswami A, Krailo M, Maibach R, et al. The Children's Hepatic Tumors International Collaboration (CHIC): novel global rare tumor database yields new prognostic factors in hepatoblastoma and becomes a research model. Eur J Cancer. 2016;52:92-101.

13 Hishiki T. Current therapeutic strategies for childhood hepatic tumors: surgical and interventional treatments for hepatoblastoma. Int J Clin Oncol. 2013;18(6):962-8. 